\title{
A note on "A new approach for ranking fuzzy numbers based on possibility theory"
}

\author{
Alireza Sotoudeh-Anvari ${ }^{\mathrm{a}}$, Seyed Jafar Sadjadi ${ }^{\mathrm{b}^{*}}$, Seyed Mohammad Hadji Molana ${ }^{\mathrm{a}}$ and Soheil \\ Sadi-Nezhad ${ }^{\mathrm{c}}$
}

${ }^{a}$ Department of Industrial Engineering, Science and Research Branch, Islamic Azad University, Tehran, Iran

${ }^{b}$ Department of Industrial Engineering, Iran University of Science and Technology, Tehran, Iran

${ }^{c}$ Department of Statistics and Actuarial Science, University of Waterloo, Waterloo, Canada

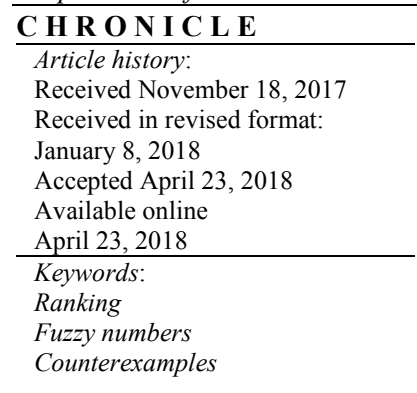

\section{A B S T R A C T}

Very recently, Qiupeng and Zuxing (2017) [Qiupeng, G., \& Zuxing, X. (2017). A new approach for ranking fuzzy numbers based on possibility theory. Journal of Computational and Applied Mathematics, 309, 674-682.] suggested a new fuzzy ranking technique on the basis of possibility theory. In this note, we show that this method leads to the incorrect result in various cases.

C) 2019 by the authors; licensee Growing Science, Canada.

\section{Background}

Comparing and ranking of fuzzy numbers (FNs) play an extremely vital role in decision-making problems under fuzzy environment. But this issue is becoming one of the key challenges in fuzzy sets. FNs are displayed by possibility distribution and can overlap with each other. Hence, it is very intricate to distinguish one FN is larger or smaller than other FNs (Kumar et al., 2011). Although numerous approaches for comparing FNs have been presented, Sotoudeh-Anvari et al. (2017) revealed that ranking of FNs is an immature operation and the majority of the suggested approaches suffer from various drawbacks.

Following these methods, very recently, Qiupeng and Zuxing (2017) proposed a new fuzzy ranking technique on the basis of possibility theory. In this note, we show that Qiupeng and Zuxing's (2017) method has an inadequacy as the same as other previous methods.

\section{Preliminaries}

To make this paper self-contained, some key concept and Qiupeng and Zuxing's (2017) method are reviewed briefly. Noteworthy, this note assumes that reader is familiar with basic concepts of fuzzy set.

* Corresponding author

E-mail address: sjsadjadi@iust.ac.ir (S. J. Sadjadi)

(C) 2019 by the authors; licensee Growing Science, Canada. doi: $10.5267 / j . d s l .2018 .5 .001$ 


\subsection{Fuzzy numbers (FNs)}

There are several kinds of FNs that can be utilized for various conditions. Owing to their theoretical and calculation ease, trapezoidal fuzzy number (TFN) is commonly used in fuzzy literature. A FN $\tilde{A}=(a, b, c, d ; w)$ is a generalized TFN if its membership function is depicted by:

$$
\mu_{A}(x)= \begin{cases}\frac{w(x-a)}{b-a} & a \leq x<b \\ w & b \leq x \leq c \\ \frac{w(x-d)}{c-d} & c<x \leq d \\ 0 & \text { otherwise }\end{cases}
$$

In Model (1), $a, b, c, d \in \mathbb{R}$ (the real number set) and $0<w \leq 1$. Also the image (opposite) of a $\mathrm{FN}$ as $\tilde{A}=(a, b, c, d ; w)$ can be obtained by $-\tilde{A}=(-d,-c,-b,-a ; w)$. Furthermore, a FN $\tilde{A}=(m, n, \alpha, \beta ; w)_{L R}$ is an $\mathrm{L}-\mathrm{R}$ form generalized FN, where $n \geq m, \alpha \geq 0, \beta \geq 0$. In this form, $\alpha$ is the left spread and $\beta$ is the right spread. The $\mathrm{L}-\mathrm{R}$ FN becomes the TFN with $a=m-\alpha, b=m, c=n$ and $d=n+\beta$.

\subsection{Qiupeng and Zuxing's (2017) method}

In the Qiupeng and Zuxing's (2017) method, $\operatorname{Mag}(\tilde{A})$ is employed to compare FNs as follows: where for a generalized FN:

$$
\begin{aligned}
& \operatorname{Mag}(\tilde{\mathrm{A}})>\operatorname{Mag}(\tilde{B}) \operatorname{Iff} \tilde{A} \succ \tilde{B} \\
& \operatorname{Mag}(\tilde{\mathrm{A}})<\operatorname{Mag}(\tilde{B}) \operatorname{Iff} \tilde{A} \prec \tilde{B} \\
& \operatorname{Mag}(\tilde{\mathrm{A}})=\operatorname{Mag}(\tilde{B}) \operatorname{Iff} \tilde{A} \sim \tilde{B} \\
& \operatorname{Mag}(\tilde{A})=\operatorname{M}(\tilde{\mathrm{A}})+\delta(\tilde{\mathrm{A}})
\end{aligned}
$$

In Model (3), $M(\tilde{A})$ is average value and $\delta(\tilde{\mathrm{A}})$ is the degree of deviation from $M(\tilde{A})$. For a generalized L-R FN $\tilde{A}=(m, n, \alpha, \beta ; w)_{L R}$ we have:

$$
\operatorname{Mag}(\tilde{A})=\frac{3 m+3 n-\alpha+\beta}{6}+\sqrt{\frac{(n-m)^{2}}{4}+\frac{(n-m)(\alpha+\beta)}{6}+\frac{(\alpha+\beta)^{2}}{24}} w
$$

\section{Counterexamples}

The following counterexamples demonstrate that Qiupeng and Zuxing's (2017) method is not correct in general.

Example 1: Consider the two generalized L-R FNs $\tilde{A}=(5,6,2,2 ; 1)$ and $\tilde{B}=(5,6,2,2 ; 0.8)$ adopted from Qiupeng and Zuxing (2017). By Qiupeng and Zuxing's (2017) method, the ranking of these FNs is $\tilde{A} \succ \tilde{B}$. Therefore, we logically derive that the ranking of the images of these FNs as $-\tilde{A} \prec-\tilde{B}$. But according to Qiupeng and Zuxing's (2017) method, the ranking of images is $-\tilde{A} \succ-\tilde{B}$. As can be seen, Qiupeng and Zuxing's (2017) approach fails to rank these FNs acceptably. This shortcoming can be 
investigated in comparing various pairs of L-R FNs as $\tilde{A}=(m, n, \alpha, \alpha ; w)$ and $\tilde{B}=\left(m, n, \alpha, \alpha ; w^{\prime}\right)$ (it can be simply checked). Also in this example, the ranking of FNs does not fulfill the reasonable property (Wang and Kerre, 2001), namely $\tilde{A} \succ \tilde{B} \Rightarrow \tilde{A} \odot \tilde{B} \succ \tilde{B} \ominus \tilde{B}$.

Example 2: Consider the two L-R FNs $\tilde{A}=\left(1,1,0,0, w_{1}\right)$ and $\tilde{B}=\left(1,1,0,0, w_{2}\right)$. According to Qiupeng and Zuxing's (2017) method, the ranking is $\tilde{A} \sim \tilde{B}$. This result is rational and satisfies Wang and Kerre's (2001) principles. However, this outcome is independent of the height of FNs and in other examples of the Qiupeng and Zuxing's (2017) paper the ranking of FNs depends on the height of FNs. Clearly, it is a contradiction.

Example 3: Consider the two L-R FNs $\tilde{A}=(4,4,1.8,2 ; 0.9)$ and $\tilde{B}=(4,4,1.8,2 ; 1)$ adopted from Qiupeng and Zuxing (2017). By Qiupeng and Zuxing's (2017) method, the ranking of the FNs is $\tilde{A} \prec \tilde{B}$ . Thus, we logically conclude that the ranking of the images of theses FNs as $-\tilde{A} \succ-\tilde{B}$. However, on the basis of Qiupeng and Zuxing's (2017) method, the ranking of images is $-\tilde{A} \prec-\tilde{B}$. Moreover, in this case, we have $\tilde{B} \succ \tilde{A} \not \nexists \tilde{B} \ominus \tilde{A} \succ \tilde{A} \odot \tilde{A}$. On the other words, Qiupeng and Zuxing's (2017) approach fails to acceptably compare these FNs again.

Example 4: Consider the two TFNs $\tilde{A}=(0.1,0.3,0.3,0.5 ; 0.9)$ and $\tilde{B}=(0.2,0.3,0.3,0.4 ; 0.9)$. By Qiupeng and Zuxing's (2017) method, the ranking is $\tilde{A} \prec \tilde{B}$. As a result, we logically infer that the ranking of the images of FNs as $-\tilde{A} \succ-\tilde{B}$. However, according to Qiupeng and Zuxing's (2017) method, the ranking of images of these FNs is $-\tilde{A} \prec-\tilde{B}$. On the other words, this technique is not able to correctly order FNs in this example again.

\section{Discussion}

Recently, Sotoudeh-Anvari et al. (2017) proposed various suggestions for verification of fuzzy ranking techniques. However, none of them was investigated in Qiupeng and Zuxing's (2017) paper. For example, a new ranking method must be justified by reasonable properties proposed by Wang and Kerre (2001) for ranking of FNs. Considering $\tilde{A}_{1}=(4,4,1.8,2 ; 0.9), \tilde{A}_{2}=(4,4,1.8,2 ; 1)$, $\tilde{A}_{3}=(4,4,3,1 ; 0.8), \quad \tilde{A}_{4}=(4,4,1,3 ; 0.8)$ in "Example 1" of Qiupeng and Zuxing's (2017) paper, the authors claimed that "intuitively" the ranking is $\tilde{A}_{4} \succ \tilde{A}_{2}>\tilde{A}_{1}>\tilde{A}_{3}$. Nevertheless, without any evidence on the basis of Wang and Kerre's (2001) study, it is nothing except a personal judgment. Moreover, the discrimination power of a fuzzy ranking technique depends on its capability to rank FNs with overlap. However, FNs in the majority of numerical examples of Qiupeng and Zuxing's (2017) paper (for example, Example 4) have no strong overlap with each other (Sotoudeh-Anvari et al., 2017). More formally, although outcomes of Qiupeng and Zuxing's (2017) technique in some cases are reasonable, it is not because of discrimination power of this technique.

\section{Conclusion}

Although no fuzzy ranking technique is fully ordered, Qiupeng and Zuxing (2017) reported that their approach is proven to be an efficient and potential alternative to the other ranking approaches. But they provided no proof for these claims. Hence, in this note some inadequacies of Qiupeng and Zuxing's (2017) method were revealed. 


\section{References}

Kumar, A., Singh, P., Kaur, A., \& Kaur, P. (2011). A new approach for ranking nonnormal p-norm trapezoidal fuzzy numbers. Computers \& Mathematics with Applications, 61(4), 881-887.

Qiupeng, G., \& Zuxing, X. (2017). A new approach for ranking fuzzy numbers based on possibility theory. Journal of Computational and Applied Mathematics, 309, 674-682.

Sotoudeh-Anvari, A., Sadjadi, S. J., \& Sadi-Nezhad, S. (2017). Theoretical Drawbacks in Fuzzy Ranking Methods and Some Suggestions for a Meaningful Comparison: An Application to Fuzzy Risk Analysis. Cybernetics and Systems, 48(8), 551-575.

Wang, X., \& Kerre, E. E. (2001). Reasonable properties for the ordering of fuzzy quantities . Fuzzy sets and systems, 118(3), 375-385.

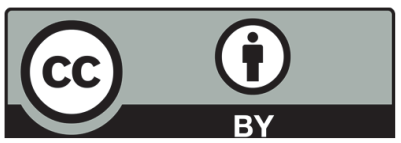

(C) 2019 by the authors; licensee Growing Science, Canada. This is an open access article distributed under the terms and conditions of the Creative Commons Attribution (CC-BY) license (http://creativecommons.org/licenses/by/4.0/). 